\title{
La riviera de Rimini : ses plages et ses "bagni"
}

Un cumul d'urbanité

\section{Thérèse Rouleau-Racco}

\section{(2) OpenEdition}

\section{Journals}

Édition électronique

URL : http://journals.openedition.org/tourisme/138

DOI : 10.4000/tourisme.138

ISSN : 2492-7503

Éditeur

Éditions touristiques européennes

Édition imprimée

Date de publication : 1 juin 2014

Pagination : 42-57

ISSN : 2109-5671

Référence électronique

Thérèse Rouleau-Racco, "La riviera de Rimini : ses plages et ses "bagni" », Mondes du Tourisme [En ligne], 9 | 2014, mis en ligne le 15 septembre 2015, consulté le 14 novembre 2019. URL : http:// journals.openedition.org/tourisme/138; DOI : 10.4000/tourisme.138

\section{c) (i)}

Mondes du tourisme est mis à disposition selon les termes de la licence Creative Commons Attribution - Pas d'Utilisation Commerciale - Pas de Modification 4.0 International. 


\title{
La riviera de Rimini :
}

\section{ses plages et ses "bagni" Un cumul d'urbanité}

\author{
ThÉrèse Rouleau-Racco \\ Doctorante en géographie \\ Université d'Angers, UFR ESTHUA, ESO-Angers (UMR CNRS 6590) \\ [therese.racco@etud.univ-angers.fr]
}

Résumé. Le concept d'urbanité est au cœur de la recherche sur les lieux touristiques. Les plages mises en tourisme, comme tout lieu touristique, ont une urbanité singulière. Ce sont les individus qui en les choisissant pour leur projet de recréation configurent les lieux et y font émerger les attributs de l'urbanité. Les plages de la riviera de Rimini et leur succession d'établissements balnéaires, communément appelés "bagni", fournissent un terrain de choix pour l'étude de ce processus. Le cas étudié illustre aussi comment l'accumulation d'urbanité, par la diversification des pratiques touristiques, l'agencement spatial et les stratégies d'acteurs, permet à un lieu touristique de continuer à fonctionner par et pour le tourisme.

Abstract. The concept of urbanness is at the heart of research on touristic locations. Tourist beaches, like any other tourist place, have their own particular urban characteristics. When people choose them for recreation, they shape these spaces and bring out urban attributes. The beaches of the Rimini Riviera and their series of beach clubs commonly known as "bagni" - provide an excellent case study to better understand this process. This case also illustrates how the accumulation of urbanness, through the diversification of tourist practices, spatial organization and stakeholders' strategies, allow a tourist place to continue operating by and for tourism. 
a plage est un site touristique (Équipe Mit, 2002 ; Duhamel, 2003), un lieu inventé, c'està-dire créé "par le regard et l'usage des touristes" (Stock, 2003, p. 287), "un lieu dans lequel les touristes passent, mais ne séjournent pas" (Équipe Mit, 2002, p. 221). Plusieurs travaux (Duhamel et Knafou, 2003 ; Coëffé, 2007, 20 I 0a, 20 I Ob) ; Coëffé et Violier, 2008 ; Rieucau, 2008) ont montré que la plage, à l'instar des autres lieux touristiques, s'inscrit dans "les mondes urbains du tourisme" (Duhamel et Knafou, 2007). Mais, si l'urbanité de la plage s'exprime de manière singulière (Coëffé, 2008, 20 I0a, 20 I0b), elle n'en est pas moins urbaine pour autant.

Le concept d'urbanité est au cœur de la recherche sur les lieux touristiques, et plusieurs travaux ont montré qu'il permet de mieux cerner la nature et la qualité de ces lieux (Coëffé et Violier, 2008 ; Équipe Mit, $201 \mathrm{I}$; Stock et Lucas, 20 I2). On peut donc penser que, à l'instar de tout autre lieu touristique, c'est l'accumulation d'urbanité qui permet aux plages mises en tourisme de durer et que c'est le concept d'urbanité qui permet le mieux, à un moment de leur histoire, d'appréhender la qualité des lieux.

C'est ce que souhaite montrer cet article en s'intéressant plus spécifiquement aux plages italiennes de la province de Rimini. Comme la plupart des plages des grandes régions touristiques de l'Italie du Nord et du Centre ${ }^{(1)}$, elles sont largement occupées par des établissements balnéaires, les "bagni" comme on les appelle communément en Italie. Près de $37 \%$ des Italiens qui séjournent à la mer fréquentent un établissement balnéaire, près de $71 \%$ d'entre eux en Émilie-Romagne ${ }^{(2)}$. La présence de ces établissements se matérialise par des forêts de parasols parfaitement alignés, des rangées de cabines, des successions de terrains de beachvolley et une accumulation de jeux pour enfants aux couleurs chatoyantes. La riviera de Rimini, nom touristique donné aux cinq communes littorales ${ }^{(3)}$ de la province, est un terrain privilégié pour approfondir les attributs urbains de la plage, s'interroger sur le processus par lequel "l'urbain produit le touristique, et le touristique produit de nouvelles urbanités" (Stock et Lucas, 2012, p. 29) et sur le lien entre la qualité touristique des lieux et les modalités de leur urbanité.

Une recherche documentaire sur l'histoire des bains de mer en Italie, et celle de ces plages en particulier, a constitué le point de départ de notre étude. L'analyse des sites internet des "bagni", des observations (participantes et non participantes), effectuées au cours de l'été 2012, ainsi que neuf entretiens semi-directifs avec des plagistes et des professionnels du secteur, nous ont permis de compléter notre recherche d'informations. Nous avons par ailleurs réalisé trente-sept entretiens semidirectifs, de juillet à septembre 2012, auprès d'un échantillon d'hébergements, avec l'objectif de cerner comment les touristes "habitaient" ce littoral, leurs "arts de faire" (Certeau, 1990) avec les lieux ; une rubrique du guide d'entretien portait plus particulièrement sur la façon de vivre la plage.

Nous analyserons tout d'abord comment l'urbain a gagné les plages et ce rivage lors de leur mise en tourisme. Nous nous intéresserons ensuite aux modalités par lesquelles l'urbanité se manifeste en de tels lieux, pour montrer enfin qu'elle naît des rapports que les individus entretiennent avec les lieux, leurs pratiques, leurs modes de vie, leur créativité aussi, et comment en configurant les lieux, elle leur permet de durer.

\section{L'URBANISATION ${ }^{(4)}$ DU RIVAGE}

L'Émilie-Romagne est la première région balnéaire d'Italie, avec 21,6 \% des nuitées des "localités marines ${ }^{(5)}$ ". Au sud du delta du Pô, de Ravenne à Cattolica, un ensemble urbain continu s'étend parallèlement au trait de côte sur environ soixantedix kilomètres. La riviera de Rimini est la partie la plus méridionale de cette ville linéaire (Turri, 2000). Cet ensemble, né du tourisme, est le résultat d'une urbanisation qui s'est faite par phases successives, sans planification globale, et a peu à peu créé puis englobé différentes entités littorales dans un tissu urbain quasi continu. L'urbanisation y est structurée de manière longitudinale par le système des infrastructures : la voie ferrée, la route nationale Adriatique SS 16, jamais à plus d'un kilomètre du rivage (Turri, 2000), et en amont l'autoroute A 14 BologneTarente. La ligne ferroviaire ${ }^{(6)}$ consti- 


\section{ILLUSTRATION 1 • RimINI, LA PLATE-FORME Et LES CABINES SUR PILOTIS. \\ Photographie figurant dans un album des frères Contessi, \\ PUBLIÉ ENTRE 1892-1902}

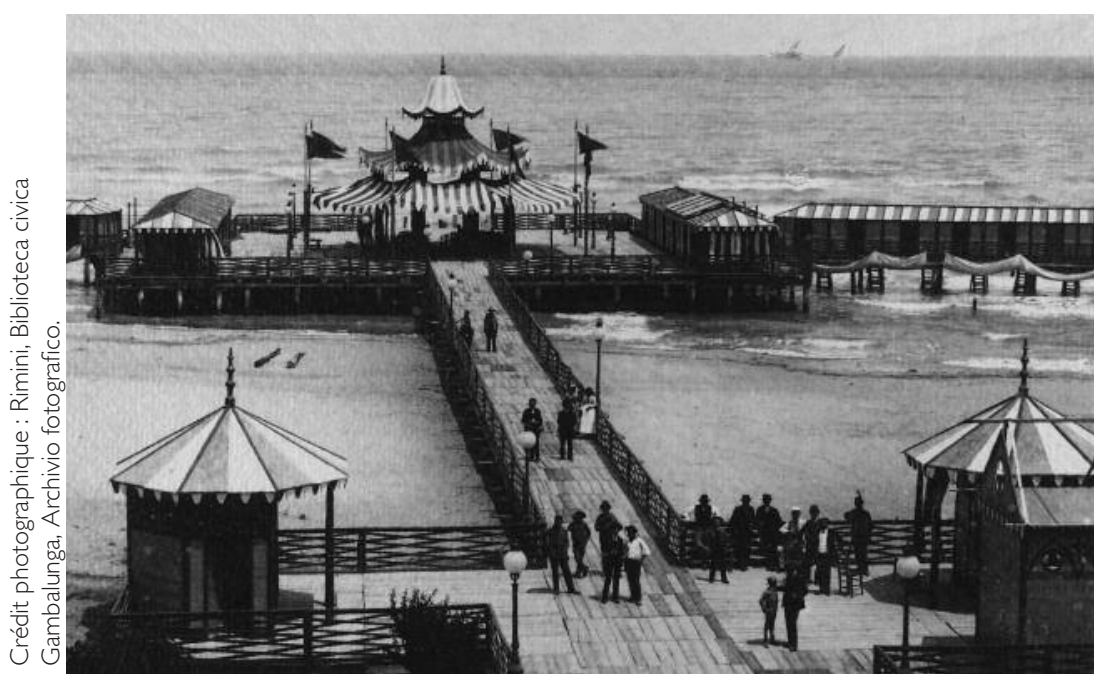

tue le plus souvent une véritable frontière physique, qui marque l'entrée dans la ville balnéaire, avec ses rues rectilignes, qui dessinent des damiers plus ou moins réguliers.

Côte basse et sablonneuse, baignée par l'Adriatique, la riviera de Rimini est un haut lieu du tourisme de masse, connu pour son tourisme balnéaire, populaire et familial. En 2012, les 170000 lits marchands de la riviera ont accueilli un peu plus de trois millions de touristes et près de 15,8 millions de nuitées ${ }^{(7)} .675$ établissements balnéaires ${ }^{(8)}$, quelques colonies de vacances et près de quarante plages publiques ${ }^{(9)} s^{\prime} y$ partagent environ trente-trois kilomètres de plage. La configuration actuelle du rivage est le résultat d'un long processus d'urbanisation, commencé il $\mathrm{y}$ a plus de cent cinquante ans.

\section{De la plate-forme au rivage : naissance de la ville balnéaire ${ }^{(10)}$}

À Rimini et dans les environs, la présence de baigneurs ${ }^{(11)}$, malgré le manque total d'infrastructures, donne l'idée à des notables locaux, d'ouvrir un établissement de bains.

La ville est située à plus d'un kilomètre en arrière du rivage et le littoral a longtemps été une zone quasi déserte, occupée par des dunes et des potagers. Ils doivent donc construire une route carrossable et mettre en place un service de navette pour relier la plage au centre urbain. Le premier établissement balnéaire de la côte adriatique voit ainsi le jour à Rimini, en juillet $1843^{(12)}$. Il s'agit d'une plate-forme en bois, équipée de cabines et montée sur pilotis, qui est reliée à la plage par une passerelle. Des escaliers permet- tent de descendre directement dans l'eau.

Cependant l'entreprise est peu rentable : la clientèle d'élite locale est trop restreinte, et Rimini n'exerce pas une attraction culturelle et récréative suffisante pour attirer l'aristocratie internationale (Battilani, 200I). Quelques années plus tard, la commune rachète l'établissement et décide de le démolir pour en construire un plus moderne. C'est à cette époque que le premier secteur de littoral commence réellement à se structurer autour des édifices du nouveau complexe balnéaire (Cabassi et Sessa, 1989). Placé sous la direction sanitaire d'un célèbre médecin hygiéniste, Paolo Mantegazza, il est constitué de la plate-forme sur pilotis, remontée au début de chaque saison (cf. illustration 1), et de deux édifices en dur : le Kursaal (1873), véritable salon mondain pour les moments d'après bains, et l'établissement hydrothérapique (1876) pour les cures marines. Pour le sociologue Giorgio Triani, l'établissement de Rimini représente un véritable "moment de lieu" (Équipe Mit, 2005) dans l'histoire balnéaire italienne : "Une situation type qui nous permet de lire et périodiser le passage de l'éphémère au stable, de la distraction épisodique, occasionnelle, à l'apparition d'un système organisé de cures et de divertissements, dont la spécialisation spatiale est la condition indispensable tout autant que le signe d'une croissante demande sociale de vacances marines ${ }^{(13)}$ " (Triani, 1988, p. 52). Dans un premier temps, le succès des bains de mer rend la 
plate-forme trop exiguë et autour d'elle, sur la plage, les cabines se multiplient (Silvestrini, 1945).

Le développement des bains de mer pose cependant le problème de l'accueil des baigneurs. L'urbanisation du littoral commence à la fin du XIX ${ }^{e}$ siècle, avec la construction de villas à proximité des établissements de bains. Puis, les premiers hôtels en bord de mer ouvrent leur porte (en 1896, à Rimini), même si jusqu'au début du XXe siècle, la capacité d'accueil reposera principalement sur les logements privés (Battilani, 200I).

Tant que le bain a eu une fonction principalement thérapeutique, la plage est restée "[...] un territoire inclassable, une ligne de confins, inhospitalière pour les pieds distingués qui devaient la traverser ${ }^{(14)}$ ”. L'utilisation des plages, leur occupation avec des éléments qui permettent d'y rester et de s'y asseoir, se met en place au fur et à mesure que croît la familiarité avec l'environnement marin, que la science médicale évolue et que de nouvelles habitudes sportives se diffusent (Triani, 1988, p. 129). Les cabines s'affranchissent de leur rôle de support à l'activité de la plate-forme et deviennent des structures autonomes. Les premières tentes et points d'ombre mobiles, inspirés des voiles des marins, des ombrelles des femmes ou des pergolas des jardins, font leur apparition. On passe ainsi progressivement du bain thérapeutique au "bain plaisir" qui invite à rester sur la plage (Triani, 1988).

On peut situer aux premières années $\mathrm{du} \mathrm{XX}^{\mathrm{e}}$ siècle les premières installations stables d'équipements balnéaires sur la plage (Studio AIR, 1987). La plage devient le centre de gravité de la vie balnéaire et, dans le même temps, se densifie.

\section{Un double processus : diffusion et densification}

Ce n'est cependant qu'entre les deux guerres que l'on peut parler de véritable développement touristique. En dix ans, Rimini multiplie sa fréquentation par quatre : de 18750 vacanciers en 1922 à 74953 en 1933 (Battilani et Fauri, 2005). De nouveaux segments de la bourgeoisie urbaine investissent le rivage l'été. La côte romagnole, avec ses longues étendues de sable en pente douce, offre aussi les conditions idéales pour l'accueil des enfants et les colonies marines voulues par le régime fasciste, en vertu du mythe de la santé et de la vigueur de la race (Cardona, 1994, cité par Corsini, 2004), se multiplient. La structure réceptive des localités se transforme, passant progressivement d'une capacité d'accueil centrée sur les résidences secondaires à une offre hôtelière de moyenne gamme.

Après la Seconde Guerre mondiale, l'évolution vers un tourisme de classes moyennes, amorcée entre les deux guerres, se confirme et la fréquentation s'internationalise. Les touristes étrangers affluent vers ce littoral $^{(15)}$, où ils trouvent ce qui constitue désormais les trois éléments fondamentaux des vacances balnéaires : la mer, le sable et le soleil. Commence alors le boom immobi- lier. Les hôtels poussent de façon incontrôlée, sur de petites parcelles, les uns collés aux autres. Sur la riviera de Rimini, on passe de 477 hôtels et pensions en 1949 à 3091 en 1967, dont la moitié sur la commune de Rimini (Battilani, 2002). Rimini s'affirme comme l'une des plus importantes destinations touristiques de la Méditerranée, et les derniers secteurs de côte, restés à l'écart, sont rattrapés par le développement touristique. Puis, la raréfaction des terrains disponibles, l'adoption par les communes côtières de plans d'urbanisme, qui tentent d'endiguer les nouvelles constructions et incitent au regroupement des hôtels, mettent un frein, au milieu des années 1960, à la fièvre immobilière (Battilani, 2002).

L'urbanisation du littoral va de pair avec la densification de la plage. À l'origine, il n'existe que quelques voies d'accès au rivage et c'est en correspondance de celles-ci que se cristallise l'occupation de la plage. C'est autour de ces sortes de "têtes de pont" que se concentre la fréquentation des touristes et que se développent les structures qui hébergent les premiers services de plage : cabines de bains, local du plagiste et point de restauration. L'urbanisation croissante des fronts de mer, la construction des lungomari ${ }^{(1)}$ multiplient les points d'accès. Progressivement, par diffusion capillaire, les établissements balnéaires occupent la majeure partie de la plage (Studio AIR, 1987). "D’un côté le plus grand nombre possible de parasols et de chaises longues, de l'autre le 
lluustration 2 • Rimini Marina centre, entrées de "bagni" (aOÛt 2012)

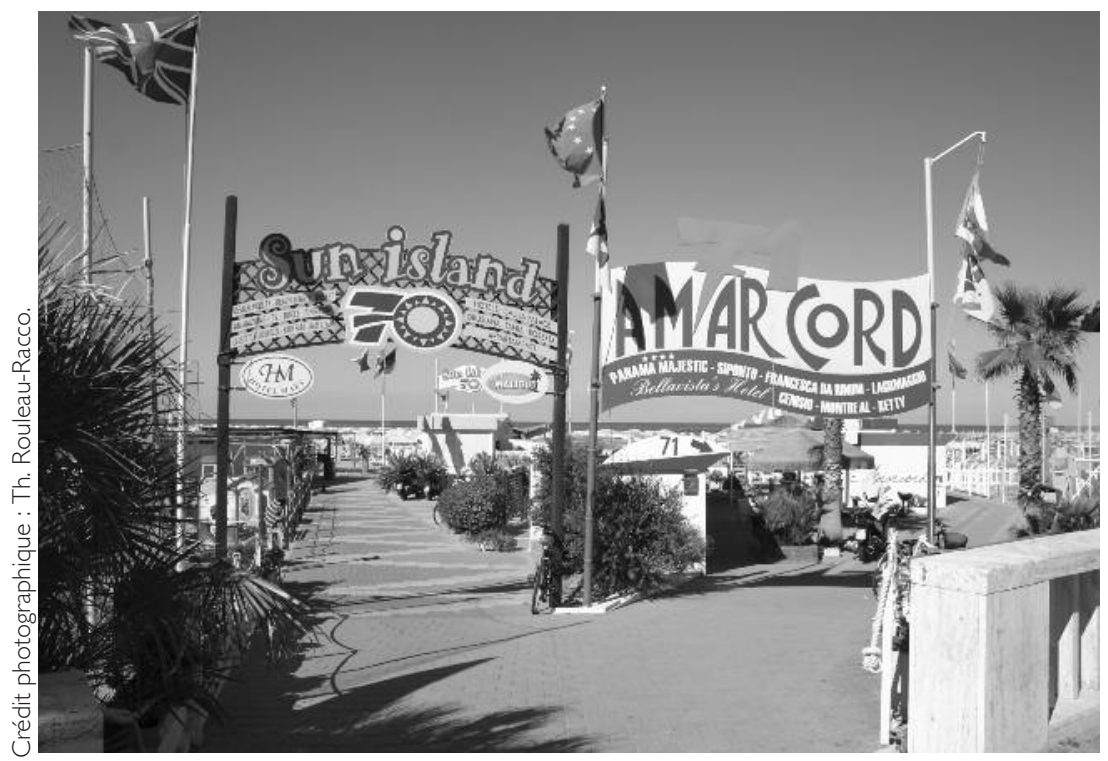

plus grand nombre de chambres ont été les concepts élémentaires et pratiques à la base de l'expansion touristique (tourisme de masse) $)^{(17)}$."

Une recherche menée à la fin des années 1980 par un cabinet d'architectes de Rimini identifie quatre phases dans l'installation d'infrastructures sur la plage : la naissance des plates-formes; l'installation sur la plage de cabines, sortes d'extensions, moins luxueuses, des platesformes ; la disparition des structures s'avançant dans la mer (platesformes et pontons) qui s'accompagne de la diffusion des établissements balnéaires directement sur la plage ; puis la substitution progressive, dans les années 1950, de ces équipements par des structures en béton, avec pour conséquence la perte du caractère saisonnier d'une partie des ins- tallations (Studio AIR, 1987). Plus de vingt ans après cette recherche, une cinquième phase est en œuvre, celle qui se joue actuellement et qui consiste à atténuer le plus possible l'impact environnemental de cette utilisation anthropique : réduction et regroupement des constructions pérennes, utilisation de matériaux naturels, panneaux photovoltaïques, etc.

\section{LA PLAGE ET SES "BAGN" : LES ATTRIBUTS URBAINS DE LA PLAGE}

L'organisation des plages de la riviera de Rimini, avec leur succession d'établissements balnéaires, interpelle $^{(18)}$. Qu'est-ce que cette organisation spatiale révèle du tourisme en ces lieux et comment, par le tou- risme, cet espace fait-il lieu ? Considérant que "l'urbain est fondamentalement à l'ouvre dans le touristique et [que] le touristique fait émerger de nouvelles formes et normes urbaines" (Nahrath et Stock, 2012, p. 9), le concept d'urbanité s'impose comme l'outil pertinent en mesure d'appréhender la qualité touristique des lieux. Mais il faut tout d'abord préciser les termes. Michel Lussault définit l'urbanité comme le "caractère proprement urbain d'un espace" et souligne qu'elle "procède du couplage de la densité et de la diversité des objets de société dans l'espace" (Lussault, 2003, p. 966). À cela Stock et Lucas (2012) ajoutent la centralité et la qualité de l'espace public tout en soulignant que l'urbanité ne relève pas seulement de la présence de ces éléments, "mais plus fondamentalement de la manière dont les individus font avec ces éléments" (Stock et Lucas, 20 I2, p. 18).

\section{Un espace dense}

Qu'il soit bordé par une promenade aménagée, par une voie de circulation ou que le bâti ne s'arrête qu'au premier grain de sable, le haut des plages est rythmé par les entrées des "bagni", qui constituent une sorte de métrique du front de mer. Chaque établissement est signalé par une enseigne qui indique le nom commercial et le numéro de l'établissement. Le numérotage en ordre croissant part en général d'une extrémité du littoral communal à l'autre ${ }^{(19)}$. La plage est ainsi subdivisée et organisée selon un système qui permet aux tou- 
ristes de se repérer et aux services urbains (premiers secours, services administratifs...) de pouvoir intervenir plus facilement. Ce système, qui transpose à la plage le système d'adressage existant dans les villes, témoigne d'entrée de jeu de son niveau d'intégration dans l'organisation urbaine (cf. illustration 2).

Le caractère urbain de cette plage ne peut échapper au regard et c'est justement la limitation du regard qui le rend si évident. La mise en tourisme de la plage a progressivement conduit à l'édification de nombreuses structures, pérennes et non, qui forment désormais un écran visuel, rendant la vue de la mer depuis le haut de plage souvent difficile, voire parfois impossible. Plus évidents en été, les marqueurs spatiaux ne disparaissent pas complètement en hiver et, même lorsque la plage est dépouillée des structures temporaires - pergolas, jeux pour enfants, parasols -, il reste en haut de plage les constructions en durcabines, bars, locaux des plagistes - auxquelles s'ajoutent les palissades de protection contre les tempêtes.

La profondeur de la plage, la densité et la taille des établissements balnéaires varient selon les secteurs de côte. Autant de facteurs qui ont influencé la disposition actuelle des constructions, figée par l'arrivée du béton. Partant de l'agencement spatial qui s'est consolidé au fil du temps, le "plan plage" (piano spiaggia) de chaque commune, sorte de plan d'urbanisme spécifique à la plage, tente de réglementer et de rationaliser l'organisation de l'espace. Quelles que soient les variantes communales, l'établissement balnéaire est toujours aménagé en zones fonctionnelles ( $c f$. illustration 3).

En haut de plage, la zone "filtre", entre le front de mer et la plage, correspond à l'entrée de l'établissement avec le parking pour les cycles; elle comporte la plupart du temps une zone de végétation, avec souvent l'utilisation d'essences visant à conférer une touche d'exotisme au lieu. Puis, c'est la zone où se concentrent les infrastructures de services : local des plagistes, sanitaires, terrains de jeux, pergolas pour les activités ludiques et festives et souvent aussi un bar-restaurant. Dans la partie médiane, s'alignent les parasols - parfois, comme à Riccione, les "tentes" (le tende), sortes de pergolas des premiers temps touristiques; c'est la zone de séjour, la zone de l'ombre souvent bordée, côté mer, par une zone solarium, où prennent place les transats sans parasol. Là s'arrête l'espace des "bagni". Au-delà, la bande de libre transit doit rester vide de tout équipement, exception faite des emplacements des loueurs d'embarcations et des infrastructures nécessaires à la surveillance de la baignade et au sauvetage en mer : miradors, chaises hautes, barques $^{(20)} \ldots$ De longs chemine- 
ments permettent de se déplacer à l'intérieur des établissements balnéaires : parallèles au rivage en haut de plage, pour passer d'un établissement à l'autre, et perpendiculaires pour rejoindre la mer.

L'organisation spatiale des "bagni" n'est pas sans rappeler celle de la ville. Elle délimite des zones de circulation (parkings pour vélos, cheminements), des zones privées, réservées à l'intime (le parasol ou la pergola), et des zones de socialisation où prévalent rencontres et interactions (les terrains de jeux, les aires d'animation). Les "bagni" disposent par ailleurs d'aménités typiquement urbaines - illuminations, sonorisation, système d'évacuation d'eau, téléphone, voire parfois wifi - et ont produit sur ce littoral des modes collectifs de gestion de services ${ }^{(21)}$, telle l'organisation de la surveillance de la baignade.

En fait, il s'est passé sur la plage ce qui s'est passé sur le rivage. L'arrivée des touristes a provoqué "concentration et diversité de personnes, de bâti, de services" (Duhamel, 2003, p. 50).

\section{Normes et régulations urbaines}

L'expansion à la plage du système urbain se manifeste aussi de manière beaucoup plus immatérielle, mais non moins structurante, à travers la constitution d'un appareil normatif complexe, qui organise l'espace et en réglemente l'usage.

Si l'ordre légal n'a pas attendu l'invention du tourisme pour investir la plage, son utilisation touristique a largement contribué à le diversifier et à le complexifier. Le domaine public maritime est régi par le Code civil, le code de la navigation et un ensemble de lois. Sa gestion administrative a été transférée de l'État aux régions (décret législatif 112/98), qui ont dû légiférer afin de réglementer leurs nouvelles attributions. En ÉmilieRomagne, la loi date du 31 mai 2002 et délègue certaines fonctions aux communes littorales ; la gestion des concessions de plage notamment.

En fait, l'appareil normatif spécifique à la plage traduit les différents enjeux d'un même espace. La plage est tout d'abord un bien public, dont la concession à des privés entraîne la mise en place de règles et le paiement d'une redevance. La plage est aussi un espace urbain et urbanisé, dont les collectivités territoriales, dans le cadre d'un projet global d'aménagement, fixent les règles d'utilisation du sol. C'est l'objet du plan d'urbanisme spécifique à la plage (piano spiaggia ou piano dell'arenile), mis en place par chaque commune après avis de la région. Enfin, la plage est un lieu touristique et de loisirs, dont les pouvoirs publics fixent les règles du vivre ensemble avec la publication, au début de chaque saison, de trois arrêtés balnéaires ${ }^{(22)}$ (ordinanze balneari) : l'arrêté régional, l'arrêté municipal et celui de la capitainerie. Ce cadre s'est récemment encore complexifié. À l'imbrication des compétences entre l'État et les collectivités territoriales sont venues s'ajouter les normes et directives de la Communauté européenne, ce qui d'ailleurs remet en question le mode d'assignation des concessions de plage ${ }^{(23)}$.

Le cadre normatif a aussi pour mission de réguler la fonction économique de la plage. Car si la plage n'est plus le territoire du pêcheur (Urbain, 1994), elle n'en a pas pour autant perdu sa fonction économique. À l'activité traditionnelle de pêche, désormais cantonnée dans les ports, se sont substituées des activités liées au tourisme. La plage est d'ailleurs un espace commercial convoité, que se partagent les activités jouissant de concessions (notamment les "bagni", les bars et restaurants de plage ou les locations d'embarcations) et un ensemble d'autres activités commerciales autorisées, réglementées par les arrêtés régional et communal : les écoles de voile ou de natation, la vente de glaces et de boissons, etc. À cela s'ajoute tout le commerce abusif, qui constitue un véritable casse-tête pour les pouvoirs publics $^{(24)}$. La plage avec ses "bagni" constitue une sorte de microsystème économique coproduit par des logiques d'acteurs : les touristes d'abord, pour lesquels elle est le lieu privilégié des pratiques balnéaires ; les plagistes et autres entrepreneurs, dont elle est l'outil de travail et la source de revenus; et les pouvoirs publics, qui en régulent le fonctionnement. La mise en tourisme a réinventé la plage (Urbain, 1994) et, ce faisant, elle en a densifié et diversifié tant l'espace physique que social. 


\section{Espace public, espace commun}

(Lussault, 2003)

L'occupation de la plage par une succession de concessions privées pose cependant question. Peut-on encore parler d'espace public ? Audelà du débat sur sa privatisation ${ }^{(25)}$, c'est la question de la qualité de l'espace public comme attribut de l'urbanité des lieux qui se pose ici ; la qualité d'espace public urbain en tant qu'espace accessible, disponible pour que les individus les plus divers puissent, dans l'anonymat, s'y rencontrer et interagir.

La loi italienne ${ }^{(26)}$ affirme le droit de chacun au libre accès à la mer. Toute personne doit donc pouvoir traverser librement et gratuitement un établissement balnéaire pour rejoindre la bande de libre transit (la battigia), située en bas de plage. Cette règle, en Émilie-Romagne du moins, semble généralement bien respectée.

Si l'accès aux équipements proposés par les établissements balnéaires est tarifé, les “bagni” de ce littoral ne sont pas pour autant des lieux exclusifs. L'ouverture se manifeste tant par l'absence de clôture, la possibilité de les traverser librement que par leur mode de fonctionnement, qui favorise un certain mélange des populations. Ce ne sont pas en effet des enclaves réservées aux touristes logeant dans tel ou tel hébergement, comme ce peut être le cas pour les plages intégrées à des complexes touristiques. Les “bagni” sont ouverts à tous ${ }^{(27)}$. Si la plupart des établissements ont des accords commerciaux avec des hébergements, garantissant ainsi des tarifs préférentiels aux clients de ces structures, quiconque peut fréquenter l'établissement de son choix. En leur sein se mélangent résidents temporaires, séjournant dans des hébergements marchands ou non, résidents permanents et excursionnistes ; surtout le weekend, lorsque les locaux viennent plus nombreux profiter de la plage. Selon le rapport national sur les entreprises balnéaires (SIB-Mercury, 2007), dans le Nord-Est italien Frioul-Vénétie Julienne, Vénétie, Émilie-Romagne - les abonnements à la saison représentent près de $29 \%$ des clients des établissements balnéaires. L’étude souligne d'ailleurs la tendance générale à l'augmentation des excursionnistes et des résidents, indice d'une double utilisation touristique et de loisirs.

Le mode de fonctionnement des "bagni" vise plus à l'intégration qu'à l'exclusion. Certains professionnels, avec l'appui des institutions et de la société civile, se sont dotés d'équipements particuliers pour la baignade des personnes handicapées. D'autres ont aménagé des emplacements afin de permettre aux propriétaires de chiens, normalement interdits, de profiter de la plage accompagnés de leur animal. On peut certes y voir une simple logique économique et l'exploitation de niches commerciales. Il reste que cela participe de l'ouverture des lieux et offre un service à des individus pour qui l'accès à la plage est souvent problématique, voire impossible.
Ni enclaves, ni parcs fermés, la plupart des "bagni" de ce littoral fonctionnent pour le plus grand nombre et grâce à lui. Ils permettent la coprésence d'individus dont le renouvellement constant, l'âge, les origines géographiques ou sociales ${ }^{(28)}$ garantissent la diversité. L'inscription de ces plages dans l'espace public révèle le subtil équilibre - public/privé, ouverture/fermeture, inclusion/exclusion - qui est au cœur même du fonctionnement des lieux touristiques.

Tout en rendant lisible les lieux et les services offerts ${ }^{(29)}$, l'organisation des "bagni" en zones fonctionnelles dessine une géographie des corps et des pratiques qui révèle leur urbanité singulière. Les cheminements qui traversent horizontalement et perpendiculairement les établissements sont les axes de déambulation, où quiconque peut circuler librement. En partie haute, là où se trouvent les terrains de jeux et de sport, prévaut la position debout. Le mouvement des joueurs cohabite avec l'immobilité des spectateurs, clients de l'établissement ou simples passants. C'est la zone de convivialité où se cristallise la vie sociale du "bagno". Dans la partie médiane, occupée par les rangées de parasols, les corps s'immobilisent. Le sociologue Nicolò Costa (1988) évoque la métaphore en miniature des appartements citadins, dont l'ensemble forme une sorte d'immeuble. Mais si les "bagni" avec leurs parasols, comme les immeubles avec leurs appartements, sont des territoires où l'on doit s'identifier pour être admis, sur la plage l'identifi- 
Illustration 4 - Plage de Misano Adriatico : la bande de libre transit,

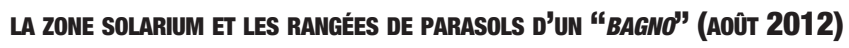

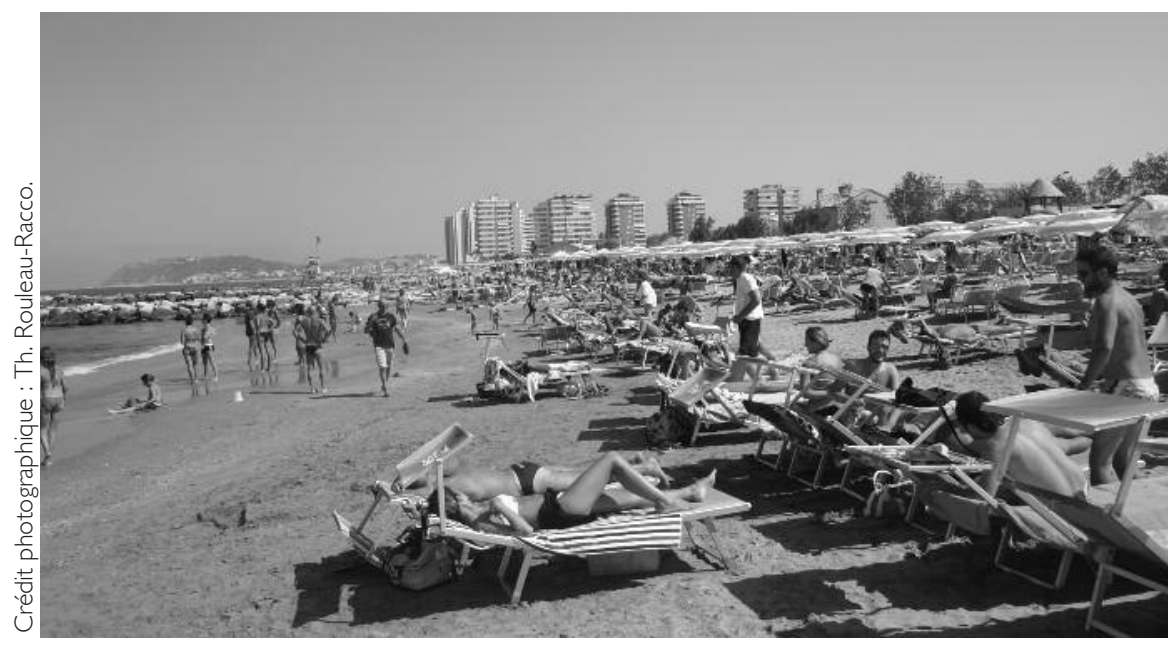

cation territoriale est moins forte, et l'espace privé exposé au regard de tous. Enfin, en bas de plage, la zone solarium est une zone d'articulation entre le domaine privé des "bagni" et la bande de libre transit, partie publique de l'estran. Là, le domaine privé est réduit à sa plus petite unité spatiale, celle du transat. L'immobilité des corps exposés au soleil côtoie le mouvement : celui des enfants qui jouent au bord de l'eau, celui des passants qui arpentent la plage dans un sens et croisent ceux qui le font dans le sens inverse, celui des regards aussi ( $c$ f. illustration 4).

Il faut sans doute, comme nous y incite Michel Lussault, dépasser le concept d'espace public et considérer ces plages comme un espace commun "un espace de convergences et d'actes, au sens où des individus y convergent [...] et y agissent, et interagissent avec les autres individus, mais aussi avec des objets, des formes spatiales" qui renvoie à trois "relations de contrariété": privé/public, intime/“extime" et individuel/social (Lussault, 2003, pp. 334-335) ; comme un espace commun produit par et pour les pratiques "recréatives" des individus (Équipe Mit, 2002).

\section{Diversité des pratiques ET PLURALITÉ DE SERVICES : LA DYNAMIQUE URBAINE DE LA PLAGE}

Appréhender la qualité des lieux touristiques, c'est cerner la qualité de leur urbanité, à travers l'organisation spatiale, mais surtout à travers le rapport que les individus entretiennent avec les lieux (Stock et Lucas, 20।2). Car ce sont les individus qui, à travers leurs pratiques, transfèrent aux lieux l'urbanité dont ils sont porteurs (Coëffé et Violier,
2008). Pour comprendre comment ces plages font lieu, il nous faut approfondir la façon dont les individus les "habitent" et donc repartir de leur projet touristique et de la manière dont ils le mettent en œuvre.

\section{Des interfaces pour se recréer}

Dans les sociétés occidentales, le choix d'une destination balnéaire coïncide généralement avec un projet de recréation centré sur le repos (Mondou et Violier, 2009). La plage en général et l'établissement balnéaire en particulier est le lieu où ce projet prend corps, au propre comme au figuré. En italien, les transats des "bagni" s'appellent d'ailleurs lettini, littéralement "petits lits".

Pour pouvoir s'installer dans un établissement balnéaire, il faut louer un "équipement" - un parasol avec un ou plusieurs lettini ou bien un simple lettino -, ce qui donne accès à un certain nombre de services : douches, vestiaires, mais aussi jeux pour enfants, animations...

Selon une enquête réalisée pour un syndicat professionnel ${ }^{(30)}$, en Émilie-Romagne, les personnes fréquentent un établissement balnéaire pour le confort (30\%), pour la large gamme de services qu'ils y trouvent $(26 \%)$ et pour la sécurité $(19 \%)$ : hygiène, surveillance, etc. On peut donc considérer que les "bagni" jouent en quelque sorte un rôle d'interface, qu'ils sont un dispositif qui ménage l'altérité (Gay, 2008). Ils libèrent les vacanciers des contingences matérielles, à commencer par celle de devoir trouver une place sur une plage fréquentée, 
et permettent aux individus de vivre la plage avec le confort et les usages auxquels ils sont habitués dans leur quotidien.

Cette fonctionnalité des "bagni" a trois effets principaux. La plage est “désensauvagée” (Urbain, 1994), aseptisée et aménagée. Le sable est nettoyé, les algues et autres détritus de laisse ramassés, des poubelles pour le tri sélectif des déchets sont installées en différents points, etc. Le deuxième effet tient à l'instauration d'un cadre sécurisé et sécurisant, à même de transmettre une sensation de tranquillité, tant physique que mentale. Le dispositif de surveillance de la baignade est très contraignant ( $c f$. note 20 ) et le vivre ensemble encadré par des règles formelles et formalisées ${ }^{(31)}$. En fait, les normes de comportement "sont plus explicites et formelles (et donc perçues comme contraignantes) si 'la' plage n'est pas un bien commun, accessible à tous les touristes, mais 'un' segment de territoire privatisé $e^{(32)}$ [...]" (Costa, 1988, p. 207). À cela s'ajoute la présence physique et bienveillante du gestionnaire, le "bagnino", qui veille au bon fonctionnement des lieux et à la satisfaction de ses clients. L'autre aspect, mais non le moindre, tient au régime d'urbanité propre à la plage (Coëffé, 2010b). La dénudation contribue à donner une impression de liberté et l'illusion d'un certain égalitarisme. Mais la proximité des corps dénudés entraîne aussi la mise en tension des normes de civilités habituelles et requiert la maîtrise de certaines compétences pour gérer les inter- actions avec les autres individus (Coëffé, 2007, 20 I0b). L'organisation de l'établissement balnéaire encadre les comportements et facilite l'apprentissage des savoir-faire. Nul besoin d'expérience particulière pour se retailler un espace à soi : pas besoin de stratégies d'installation ou de savantes manœuvres pour l'annexion d'une portion de sable (Coëffé, 2007 ; Urbain, 1994). L'espace privé dévolu à chaque individu dépend des équipements loués et l'écartement entre les parasols, fixé par les arrêtés régional et communal, pose les bases des distances à respecter. Il y a cependant toujours possibilité de ruser (Certeau, 1990), comme dans la zone solarium, où les transats sans parasol sont mobiles ( $c f$. illustration 4). En outre, à l'intérieur du "bagno", tout un chacun est en permanence exposé au regard des autres et c'est toujours la compétence des individus qui orchestre le jeu des regards et la maîtrise des émotions (Coëffé, 2010b).

En fait, tel le camping, l'établissement balnéaire constitue un espace sécurisant "où l'on peut recréer son espace privé au sein d'une vie communautaire à la promiscuité choisie. Reconstituant dans des lieux hors quotidiens un univers familier et routinier [...]" (Équipe Mit, 20l I, p. 27).

\section{Diversification des services et cumul des pratiques}

Le jeu est, avec le repos, l'autre modalité par laquelle le projet de recréation des individus se concrétise
(Équipe Mit, 20I I) et l'autre axe autour duquel s'articule l'activité des "bagni”. Lorsqu'on parcourt les plages, on ne peut qu'être surpris par la diversité des équipements mis à disposition, pour la plupart gratuitement (inclus dans le prix de la location du parasol et des transats), dans un espace qui se veut naturel : terrains de beach-volley, beach-tennis, foot-volley, basket, boulodromes, tables de ping-pong, babyfoot, jeux pour enfants, mais aussi aire de fitness-musculation, piscine, bibliothèque, etc. Au-delà de la diversité, les équipements proposés s'articulent autour de deux grands types de pratiques. Le soin de soi, avec pour objectif d'atteindre un bien-être physique et psychique musculation, jacuzzi, lecture, yoga, etc. -, et toutes les modalités du jeu, qui permettent d'être avec les autres, ou plus justement d'être soi avec les autres (Urbain, 1994) : compétitions et tournois en tout genre, bals de groupes, etc.

Le cumul des pratiques (Deprest, 1997, citée par Duhamel et Violier, 2009) est sans doute ce qui permet aux "bagni" de durer. En fait, au fil du temps, les pratiques touristiques se sont diversifiées et démultipliées (Équipe Mit, 20I I). Au sein des "bagni", ce processus se matérialise de plusieurs manières : en premier lieu par un éventail d'équipements et d'activités toujours plus grand. L'arrivée des tapis roulants et autres appareils de musculation n'a pas détrôné le jeu de boules, ni celle des cours de Pilates supprimé l'aquagym. La gamme qualitative 
de l'offre s'est aussi élargie. Certains établissements s'en tiennent à une offre de services traditionnelle, se cantonnant aux équipements ludiques de base : terrains de beachvolley, jeux de boules, quelques jeux pour enfants. D'autres, en revanche, pointent sur une offre diversifiée ou sur des prestations qui ciblent des catégories de clientèle bien définies : les clients de l'hôtellerie haut de gamme, les jeunes, les propriétaires de chien, etc. Enfin, la diversification des pratiques a entraîné l'extension temporelle des activités vers les heures nocturnes (illumination des terrains de jeux, animations en soirée...) et l'arrivée de nouveaux services, qui de la ville se transposent sur le sable : activités culturelles, soins esthétiques, cours de cuisine ${ }^{(33)}$, etc. Il y a là sans nul doute un jeu subtil entre l'évolution des pratiques et la capacité des gestionnaires à percevoir les ouvertures commerciales qu'elles représentent, tant d'un point de vue strictement économique que compétitif.

Cette dynamique entre lieu, pratiques et acteurs n'est cependant pas spécifique à la plage, mais le propre de tout lieu touristique. Ce qui interpelle sur la plage, c'est la décontextualisation (Urbain, 1994) ou l'absence de liens entre certaines activités et le milieu marin. Même si, à y bien songer, il n'y a là rien de bien nouveau. La transposition de la vie sociale urbaine à l'estran existait déjà sur les plates-formes où, comme à Rimini, la zone centrale, occupée par la pagode chinoise, accueillait des concerts (Silvestrini, 1945). Des pilotis au sable, le mouvement s'est seulement démocratisé, amplifié, tant par le nombre des bénéficiaires que par la variété des activités.

Ce n'est pas tant la qualité du milieu biophysique (Équipe Mit, 20। I) de ces plages qui fonde désormais l'adéquation entre le projet des touristes et le choix des lieux, que la densité et la diversité des services offerts.

\section{Des plages de plus en plus intégrées au système urbain des localités}

Bien qu'elles ne se baignent que dans la piscine de leur hôtel, deux touristes luxembourgeoises nous expliquent pourquoi elles vont prendre le soleil à la plage. La mère : "On peut plus regarder les gens : comment ils se comportent, comment ils sont habillés. [...] À la plage, quand on est de nouveau chez notre 'bagno', autour de nous il y a toujours les mêmes personnes [...]. Ce sont des gens qu'on revoit seulement à la plage, qui ne sont pas ici dans l'hôtel, qui sont dans un hôtel là ou par là-bas. Mais on se revoit à la plage. On va aussi boire un coup au bar. On parle ${ }^{(34)}$...”

À la manière des villages de vacances, la fonction hébergement en moins, les "bagni" favorisent une sociabilité particulière. Ils constituent de petites communautés éphémères propices à la socialisation, d'autant plus que les établissements ne se contentent pas de mettre à disposition équipements et services, mais en organisent généralement la mise en scène. Tournois de boules, com- pétitions de beach-volley, bals de groupes, cours collectifs, ateliers pour les enfants, etc. renforcent la sensation d'être dans un lieu et dans un temps hors du quotidien. Cette organisation permet, au sein de la cellule familiale ou du groupe d'amis, de resserrer les liens (Urbain, 1994), mais aussi de faire de nouvelles rencontres. Les "bagni" sont en fait des lieux d'urbanité festive, des lieux de sociabilité pacifique (Urbain, 1994).

"Les établissements s'équipent pour tripler les offres, potentialiser les services en transformant les plages en nouvelles places [de village]. La mer est la toile de fond, le reste est business et créativité(3)" (Cavallieri et Scalise, 2013) : en introduisant un parallèle avec la place du village, les journalistes de La Repubblica soulignent tout à la fois la dimension communautaire, la qualité urbaine des lieux mais aussi, surtout en Italie, sa centralité sociale.

L'évolution des pratiques touristiques, leur cumul dans le temps et dans l'espace actualisent constamment les attributs urbains des plages. L'existence et la permanence des "bagni" tiennent à la dynamique entre la diversification et la multiplication des pratiques touristiques et de loisirs et un système d'acteurs locaux (gestionnaires mais aussi institutions), qui perçoit et ajuste l'offre et les règles du jeu aux évolutions des pratiques ${ }^{(36)}$. C'est cette dynamique entre le lieu, les pratiques et les acteurs qui permet aux "bagni" de durer et, ce faisant, de faire des plages de ce littoral un espace ludique et festif, toujours plus inséré 
dans le système urbain des localités. Durant quelques mois, ces plages deviennent le centre de gravité de la vie touristique. Elles constituent les lieux de coprésence maximum, où convergent des populations variées, tant par l'origine, l'âge, la condition sociale que par le rapport aux lieux : touristes, résidents, excursionnistes. À l'échelle des localités balnéaires, les plages et leur succession de "bagni" constituent, en été, un pôle recréatif et récréatif incontournable, ce qui leur confère sans nul doute des attributs de centralité (Ceriani-Sebregondi et alii, 2008).

\section{Conclusion}

Avec leur mise en tourisme, les plages sont devenues des lieux touristiques. Comme tout lieu touristique, elles sont fondamentalement des lieux urbains dont l'urbanité s'exprime de manière singulière (Coëffé, 20l0b). La qualité et l'intensité de leur urbanité est liée au processus touristique qui s'y joue.

L'étude des plages de la riviera de Rimini, majoritairement occupées par des établissements balnéaires, montre comment le concept d'urbanité permet d'appréhender la qualité d'un lieu touristique comme la plage. Leur organisation spatiale aménagement, bâti, normes urbaines, articulation entre espace privé et espace public - leur confère des attributs urbains, mais c'est principalement la manière dont les individus, qui choisissent ces plages pour leur projet de recréation, "habitent" les lieux qui produit et actualise sans cesse cette urbanité particulière.
Cette étude de cas montre aussi comment l'accumulation d'urbanité permet à un lieu de maintenir dans le temps une fonction touristique élevée. Cette accumulation résulte d'une dynamique entre diversification des pratiques touristiques, stratégies d'acteurs, agencements spatiaux, services offerts et normes développées. Et si la mise en tourisme des plages chasse le pêcheur (Urbain, 1994), elle ne dépossède pas pour autant systématiquement les habitants de leur plage (Duhamel et Violier, 2009). Elle peut aussi permettre à ces derniers de changer d'univers économique (Urbain, 1994) et d'en faire les protagonistes du développement local. Le questionnement sur l'urbanité de la plage doit donc être poursuivi, en s'interrogeant notamment sur les liens entre les modalités de l'urbanité des plages et le rôle joué par les acteurs locaux dans leur mise en tourisme.

\section{NOTES}

(*) Toutes les traductions des textes italiens ont été effectuées par nos soins.

( I) Émilie-Romagne, Vénétie, Toscane et Ligurie représentent $62 \%$ des nuitées effectuées dans les "localités marines" (catégorie définie par l'Istat, l'équivalent de l'Insee). Le littoral est un espace touristique majeur de la péninsule, dont les Italiens constituent la première clientèle (67,5 \% des nuitées). Données Istat, 2007 (ONTIT, 2009).

(2) SIB-Isnart, 2004. Enquête téléphonique portant sur 2003 individus de quinze ans et plus, résidant en Italie, qui ont effectué au moins un voyage d'agrément dans les six mois précédant l'enquête. Les résultats indiqués portent sur la population dont la destination des vacances principales se trouvait en Italie.

(3) Communes de Bellaria-Igea Marina,

Rimini, Riccione, Misano Adriatico et Cattolica.

(4) Par "urbanisation", on entend I'"action d'urbaniser, de donner les caractères de la ville; le résultat de cette action". (Définition du TLF - Trésor de la langue française, mis en ligne par le Centre national de ressources textuelles et lexicales:

[www.cnrtl.fr].)

(5) Donnée ISTAT, 2007. Rapport de I'Observatoire national du tourisme, II Turismo balneare in Italia, 2009.

(6) Au sud de Rimini, il s'agit de la voie ferrée Bologne-Ancône, mise en service en |86| ; au nord, c'est la ligne RiminiRavenne, inaugurée en 1889.

(7) Avec un parc de | 205 structures (dont I I 8 établissements hôteliers) et une capacité de 74933 lits marchands, Rimini accueille à elle seule près de la moitié de la fréquentation de la riviera: 
1,6 million de touristes pour 7,6 millions de nuitées (seuls les hébergements marchands sont comptabilisés). (Source : Provincia di Rimini, données 2012.)

(8) Camera di commercio, industria, artigianato e agricoltura di Rimini (CCIAA), 2012. (9) En 20।4, on dénombre trente-neuf plages publiques pour l'ensemble des cinq communes littorales. (Donnée élaborée à partir des informations publiées sur le site "Tourisme" de la province de Rimini :

[www.riviera.rimini.it].)

(10) Les informations contenues dans ce paragraphe et dans le suivant ont été puisées dans différents ouvrages et articles: Battilani (200I, 2002, 2008), Battilani et Fauri (2005), Berrino (20 I I), Cardellini et alii (1980), De Nicolò (2003), Farina (1995, 2003), Gardini, Gattei et Porisini (1977), Masini (1986, 1989), Silvestrini (1945), Studio AIR (1987), Triani (1988).

( I I) Cette "'balnéarisation' primitive" (Urbain, 1994) est mentionnée par de nombreux auteurs : Battilani et Fauri (2005), Berrino (20I I), Farina (1995, 2003), Silvestrini (1945), Triani (1988). Battilani et Fauri (2005) soulignent que l'habitude du bain de mer, dont témoignent les voyageurs du Grand Tour dès la fin du $X V I^{e}$ siècle, s'était perpétuée jusqu'au début du XIXe siècle et était répandue dans toutes les classes et tranches d'âge. Farina (1995) mentionne la présence d'aristocrates étrangers, dont des membres de la famille Bonaparte, venus prendre des bains à Rimini et à Cattolica.

(I2) Dans les premiers établissements de bains situés sur le littoral de la péninsule (Livourne, 1780), l'immersion dans l'eau salée se faisait dans des baignoires. Puis, à partir des années 1820, sur l'Adriatique, apparaissent des structures flottantes. Amarrées dans des bassins portuaires
(Trieste, 1823, Ancône, 1835) ou dans la lagune de Venise (I833), elles permettent une immersion directe. C'est à Viareggio (Toscane), en 1827, qu'est construite directement sur la plage la première plateforme en bois sur pilotis.

(13) "Una situazione tipo che ci consente di leggere e periodizzare il passaggio dall'effimero allo stabile, dallo svago episodico, occasionale, al sorgere di un sistema organizzato di cure e divertimenti, di cui la specializzazione spaziale ne è la condizione indispensabile oltre che il segno di un'aumentata domanda sociale di vacanze marine."

(I4) "[...] un territorio inclassificabile, una linea di confine, inospitale per i piedi signorili che dovevano attraversarla."

(I5) À Rimini, dans les années 1960, la fréquentation étrangère représente jusqu'à $40 \%$ de la fréquentation totale, alors qu'elle n'est que de $4 \%$ entre les deux guerres (Battilani et Mussoni, 2007).

(16) Littéralement "le long de la mer". Le lungomare désigne l'axe qui suit le rivage, qu'il soit piétonnier ou accessible à la circulation automobile.

(17) "Da una parte il maggior numero possibile di ombrelloni e sdrai, dall'altra il maggior numero di camere, sono stati i concetti elementari e pratici alla base della espansione turistica (turismo di massa)." (Dans Rimini e il suo territorio. Riflessioni sul futuro di Rimini [Rimini et son territoire. Réflexion sur le futur de Rimini], p. 29, document en ligne, ni daté, ni signé, à partir du site consacré au Plan stratégique de la commune de Rimini :

[www.riminiventure.it/binary/rimini_venture _new/documenti/07_RIMINI_e_il_suo_TER RITORIO. I 246445646.pdf]).

(18) La majeure partie de la superficie des plages est occupée par des concessions privées. Dans la province de Rimini, selon les communes, en 2012 , les plages publiques représentaient entre $3 \%$ et 12,5\% du littoral communal. (Source : recensement effectué en 2012 , auprès des communes littorales, par un groupe politique. Document en ligne à partir de : [http://aldemetra.regione.emiliaromagna.it/oggetti/doc/IX/OG20I2047696. pdf].) La loi régionale $n^{\circ} 9$ du 3I mai 2002 sur l'exercice des fonctions administratives en matière de domaine maritime et de zones de mer territoriales précise que les communes ne peuvent plus relâcher de nouvelles concessions si le pourcentage de plages publiques ne représente pas au minimum $20 \%$ du littoral communal.

(19) À Rimini, le littoral est divisé en deux secteurs séparés par le port-canal. II y a donc une double numérotation : l'une pour Rimini nord et l'autre pour Rimini sud.

(20) L'arrêté balnéaire (Ordinanza balneare) de la région Émilie-Romagne précise que la distance maximale entre les postes de sauvetage ne doit pas excéder cent cinquante mètres. Une tolérance de $10 \%$ existe toutefois en fonction de la morphologie du secteur côtier.

(2I) Claudio Travaglini (20।2) parle de modèle "industriel-coopératif" de la gestion des concessions de plage. II souligne que, sur ce littoral, parallèlement à une grande fragmentation des concessions, il existe une organisation industrielle, avec une pluralité de services avancés et différenciés, qui améliore les prestations fournies

(22) L'arrêté régional rappelle les principes généraux de l'utilisation de la plage et discipline le fonctionnement des établissements balnéaires : surveillance de la baignade et équipements de secours, mais aussi horaires et périodes d'ouverture, espacement entre les parasols, etc. L'arrêté municipal permet aux communes, en fonction de la spécificité de leur littoral, de modifier 
ou de préciser certaines des dispositions fixées par la région. L'arrêté de la capitainerie, quant à lui, traite principalement des questions relatives à la baignade et au sauvetage en mer.

(23) Notamment la directive relative aux services dans le marché intérieur (directive 2006/I23/CE), plus communément appelée "directive Bolkestein". La Communauté européenne a estimé que les normes relatives à l'octroi des concessions touristiques en vigueur en Italie se trouvaient être en opposition avec les principes de liberté d'établissement (article 49 - ex-article 43 du traité fondant la Communauté européenne) et avec ceux d'impartialité, de transparence et de publicité des procédures de sélection (article 12 de la directive 2006/I23/CE). Cela a valu à l'Italie d'être l'objet d'une procédure d'infraction, levée à la suite de l'adoption de la loi

217/20। I, dans laquelle le gouvernement italien s'engageait à revoir le régime des concessions dans un délai de quinze mois. Un an plus tard, la loi 228/2012 a cependant prorogé la durée des concessions jusqu'au 3 I décembre 2020.

(24) En 2013, la commune de Rimini a d'ailleurs édité un dépliant en cinq langues pour mettre en garde les touristes et demander leur collaboration dans la lutte contre le commerce des contrefaçons et les activités commerciales illicites.

(25) Le débat existe en Italie. II est notamment animé par les associations de défense de l'environnement, telles que le WWF ou Legambiente.

(26) Principe réaffirmé par la loi n² 296 du 27 décembre 2006, article premier, alinéa 251.

(27) À quelques rares exceptions près : les plages des colonies de vacances, celles de certains comités d'entreprise.
(28) L'enquête SIB-Isnart de 2004 indique les catégories socioprofessionnelles suivantes (résultats nationaux) : professions indépendantes, II, 9\% ; employés/enseignants, etc., 30,9\% ; ouvriers, 10,9\% ; femmes au foyer, II,9\% ; étudiants, 20,3 \% ; retraités, II,6 \% ; chômeurs ou autres, 2,5 \%. En Émilie-Romagne (résultats régionaux), 40,8 \% de la clientèle des établissements balnéaires sont des familles ou groupes d'amis avec enfants de moins de quinze ans, 33,4\% des familles ou groupes d'amis sans enfants ou avec enfants de plus de quinze ans, 19,4\% des couples (conjoints, amis) sans enfants et 6,4\% des personnes seules.

(29) Pour Isaac Joseph, l'accessibilité d'un espace public est lié, entre autres, "à la lisibilité de son 'mode d'emploi' [...]"' ((Isaac Joseph (dir.), Prendre place. Espace public et culture dramatique, éditions Recherches/Plan urbain, 1995, p. 13 - cité par Paquot, 2009. p. 8.

(30) Enquête réalisée par l'Istituto nazionale ricerche turistiche (Isnart) pour le Sindacato italiano balneari (SIB) : La soddisfazione dei clienti sui servizi degli stabilimenti balneari, 2004.

(3I) Les trois arrêtés balnéaires - celui de la capitainerie, celui de la région et celui de la commune - doivent être affichés dans chaque établissement.

(32) "sono più esplicite e formali (e percepite quindi in modo vincolante) se 'la' spiaggia non è un bene comune accessibile a tutti i turisti ma 'un' segmento di territorio privatizzato [...]."

(33) À Riccione, un établissement balnéaire propose d'apprendre à préparer la piadina, spécialité romagnole : sorte de galette salée épaisse, qui peut être consommée à la place du pain ou farcie comme un sandwich.

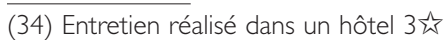
d'Igea Marina, le 6 septembre 2012.
(35) "Gli stabilimenti si attrezzano per triplicare le offerte, potenziare i servizi, trasformando le spiagge nelle nuove piazze. II mare è sullo sfondo, il resto è business e creatività."

(36) Voir à ce sujet l'étude menée par Chiara Francesconi (20I I) sur les établissements de Cervia, Milano Marittima et Pinarella.

\section{Références bibliographiques}

Patrizia BatTILANI, Vacanze di pochi, vacanze di tutti. L'evoluzione del turismo europeo, II Mulino, 200 I.

Patrizia BATTILANI, "Ascesa, crisi e riorientamento del turismo", dans Vera Negri Zamagni, Sviluppo economico a Rimini nel secondo Novecento, Istituto per la storia della resistenza e dell'ttalia contemporanea della Provincia di Rimini, Capitani Editore, 2002.

Patrizia BATTILANI, "Dal turismo balneare aristocratico a quello di massa: differenze e somiglianze riscoperte attraverso le fonti visive", Rivista di storia e storiografia on line, $n^{\circ}$ 17, 2008 [www.storiaefuturo.eu].

Patrizia BATTILANI et Francesca FAURI, "II turismo come motore dello sviluppo economico locale: il caso di Rimini", Storia del Turismo. Annale, nº 5, 2005.

Patrizia BATTILANI et Maurizio MussonI, "I turismo a Rimini: un'identità cittadina concertata", dans Lionello Franco Punzo et Stefano Usai, L'Estate al mare. Residenti e turisti in alcune destinazioni italiane, McGrawHill, 2007. 
Annunziata BerRINo, Storia del turismo in Italia, || Mulino, 20 I I.

Carlo CabAssi et Chiara SESSA, "Sulla spiaggia: architettura e attrezzature balneari", dans Lido \& lidi. Società, moda, architettura e cultura balneare tra passato e futuro, Marsilio editore, catalogue de l'exposition, Venise, juillet 1989.

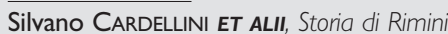
dal 1800 ai nostri giorni - Vol. VI : Vita balneare - Giomalismo - Teatro - Sport Città e campagna fra ottocento-novecento, Bruno Ghigi editore, 1980.

Maria Clelia CARDONA, La storia della villeggiatura: dall'epoca romana al Novecento,

Edizioni Abete, 1994

Marina CAVAluierI et Irene Maria SCALISE,

"Yoga alle otto, cinema al tramonto - la spiaggia ora è aperta 24 ore su 24", La Repubblica, édition du 12 août 2013.

Giorgia CerianI-Sebregondi, Amandine CHAPUIS, Jean-Christophe GAY, Rémy KNAFOU, Mathis StOCK et Philippe VIOLIER, "Quel serait l'objet d'une 'science du tourisme' ?", Téoros, vol. 27, n I, 2008.

Michel de Certeau, L'Invention du quotidien 1. Arts de faire, Gallimard, 1990 (I re édition 1980).

Vincent CoËFFÉ, "L'urbanité cachée du rivage touristique", dans Philippe Duhamel et Rémy Knafou (dir.), Mondes urbains du tourisme, Belin, 2007.

Vincent CoËFFÉ, "Le tourisme, fabrique d'urbanité. Matériaux pour une théorie de l'urbain", Mondes du Tourisme, n², 2010a. Vincent CoËFFÉ, "La plage, fabrique d'une touristi(cité) idéale", L'Information géographique, vol. 74, n³, $2010 b$.
Vincent CoËfFÉ, PhilipPe Violier, “Les lieuX du tourisme : de quel(s) paradis parle-ton? Variations sur le thème de l'urbanité touristique", Articulo-Journal of Urban Research, n³, 2008. En ligne à partir de [http://articulo.revues.org//58].

Bartolomeo CoRsinI, L'Impresa balneare Storia, evoluzione e futuro del turismo di mare, Hoepli, 2004.

Nicolò CostA, "Lo spazio sociale nello stabilimento balneare", Studi e ricerche, $n^{\circ} 2$, 1988.

\section{Maria Lucia DE Nicolò, Una marina} dell'Adriatico. Lido, arenili, bonifiche, verso la città giardino, Banca di Credito Cooperativo di Gradara, 2003.

Florence DEPREST, Enquête sur le tourisme de masse. L'écologie face au territoire, Belin, 1997.

Philippe Duhamel, "Les lieux touristiques", dans Mathis Stock (coord.), Le Tourisme. Acteurs, lieux et enjeux, Belin, 2003.

Philippe DUHAMEL et Rémy KNAFOU,

"Tourisme et littoral : intérêts et limites d'une mise en relation", Annales de Géographie, n 629, 2003.

Philippe Duhamel et Rémy KNAFOu (dir.), Mondes urbains du tourisme, Belin, 2007.

Philippe DUHAMEL et Philippe VIOLIER,

Tourisme et littoral : un enjeu du monde, Belin, 2009.

Ferruccio Farina, Una costa lunga due secoli. Storie e immagini della Riviera di Rimini, Fondazione Cassa di Risparmio di Rimini, Panozzo, 2003.

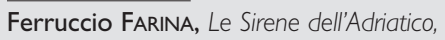
1850 - 1950, Riti e miti balneari nei manifesti pubblicitari, Federico Motta editore, 1995.
Chiara Francesconi, Di padre in figlio. Una ricerca etnosociologica sul turismo balneare romagnolo, FrancoAngeli, 201 I.

Attilio Gardini, Giorgio GatTel et Giorgio PORISINI, Storia di Rimini dal 1800 ai nostri giomi. Vol. II : Lo sviluppo economico e sociale, Bruno Ghigi Editore, 1977.

Jean-Christophe GAY, "Tourisme, interfaces et discontinuités", dans Françoise Pagney

Pénito-Espinal (dir.), Les Interfaces. Ruptures, transitions et mutations, Presses universitaires de Bordeaux, n 19, 2008.

Michel Lussault, "Espace public" et "Urbanité", dans Jacques Lévy et Michel Lussault (dir.), Dictionnaire de la géographie et de l'espace des sociétés, Belin, 2003.

Manlio MASINI, La Stagione dei bagni - Rimini nelle cronache della Belle Époque, Maggioli, 1986.

Manlio MASINI, L'Estate in camicia nera Rimini nelle cronache del ventennio, Maggioli, 1989.

Équipe MIT, Tourismes 1. Lieux communs, Belin, 2002.

Équipe MIT, Tourismes 2. Moments de lieux, Belin, 2005.

Équipe MIT, Tourismes 3. La révolution durable, Belin, 201 I.

VÉroniQue MONdOU et PHILIPPE VIOLIER,

"Projets, pratiques et lieux touristiques, quelles relations?", M@ppemonde, n 94, 22009. En ligne à partir de [http://mappemond e.mgm.fr/num22/articles/art0920l.html].

Stéphane NAHRATH et Mathis StOCK,

"Urbanité et tourisme : une relation à repenser", Espaces et Sociétés, n 151, 2012.

Thierry PAQUOT, L'Espace public, La

Découverte, 2009. 


\section{RECHERCHE • DOSSIER}

Jean Rieucau, "Vers des plages urbaines postbalnéaires au début du XXl siècle", Géographie et cultures, n 67, 2008.

Luigi SILVESTRINI, Un secolo di vita balneare al lido di Rimini 1843-1943, Azienda di

Soggiomo, 1945.

Mathis STOCK (coord.), Le Tourisme. Acteurs, lieux et enjeux, Belin, 2003.

Mathis STOCK et Léopold LuCAS, "La double révolution urbaine du tourisme", Espaces et Sociétés, $n^{\circ}$ |5|, 2012.

Studio AIR, Sulla spiaggia nascita ed evoluzione delle attrezzature balneari sulla riviera di Romagna, Maggioli, 1987.

Claudio TravaguINI, La cooperazione e lo sviluppo dei sistemi turistici locali: percorsi e ritomi in quarant'anni di sviluppo del sistema turistico riminese, Communication à la IV Réunion scientifique de la SISTUR - Société italienne de sciences du tourisme, 26-27 octobre 2012. En ligne à partir de [http://amsacta.unibo.it/337////Slstur__La_cooperazione___PAott2012.pdf].

Giorgio Triani, Pelle di luna Pelle di sole Nascita e storia della civiltà balneare 1700 1946, Marsilio, 1988.

Eugenio TURRI, La Megalopoli padana, Marsilio, 2000.

Jean-Didier URBAIN, Sur la plage, Payot et

Rivages, 1994.

\section{Études}

- CCIAA (Camera di commercio, industria, artigianato e agricoltura di Rimini), Il distretto del Turismo - Provincia di Rimini, Ufficio Studi e Statistica CCIAA Rimini, 2012. En ligne à partir de : [www.starnet.unioncamere.it/II-Distretto-Turistico-Riminese-2012_7A9910B268C353].

- ONTIT (Osservatorio nazionale del turismo) et Presidenza del Consiglio dei ministri, Il Turismo balneare in Italia, 2009. En ligne à partir de

[http://www.ontit.it/opencms/opencms/ont/it/documenti/02059?cate gory=documenti/ricerche_ONT/ricerche_mono].

- Provincia di Rimini, Osservatorio sul Turismo. Statistiques sur les capacités d'accueil et la fréquentation des hébergements marchands. En ligne à partir de

[http://www.provincia.rimini.it/informa/statistiche/turismo/2012_repo rt/index.html].

- SIB (Sindacato italiano balneari) - Isnart (Istituto nazionale ricerche turistiche), La soddisfazione dei clienti sui servizi degli stabilimenti balneari, 2004.

- SIB (Sindacato italiano balneari) - Mercury srl Turistica, Rapporto sulle imprese balneari, 2007. En ligne à partir de [http://www.fibaconfesercenti.com/iniziative/rapporto2007impresebalneari.pdf]. 